\title{
Performance of the BM@N GEM/CSC tracking system at the Nuclotron beam
}

A. Galavanov ${ }^{1,2}, M$. Kapishin $^{1}, K$. Kapusniak ${ }^{3}, V$. Karjavine $^{1}, S$. Khabarov $^{1}, Y$. Kirushin ${ }^{1}$, A. Kolesnikov ${ }^{1}$, E. Kulish ${ }^{1, *}, V$. Lenivenko ${ }^{1}, A$. Makankin $^{1}, A$. Maksymchuk $^{1}, B$. Mehl $^{3}$, R. De Oliveira ${ }^{3}, V$. Plotnikov ${ }^{1}, G$. Pokatashkin ${ }^{1}, A$. Rodriguez $^{3}, M$. Rumyantcev ${ }^{1}$, I. Rufanov ${ }^{1}, V$. Sidorenko ${ }^{1}, S$. Vasiliev ${ }^{1}$, and $A$. Vishnevskiy ${ }^{1}$

${ }^{1}$ JINR, Dubna, Russia

${ }^{2}$ MEPhI, Moscow, Russia

${ }^{3}$ CERN, Geneva, Switzerland

\begin{abstract}
BM@N (Baryonic Matter at the Nuclotron) is a fixed target experiment aimed to study nuclear matter in the relativistic heavy-ion collisions at the Nuclotron accelerator in JINR. The BM@N tracking system is based on Gas Electron Multipliers (GEM) detectors mounted inside the BM@N analyzing magnet. The Cathode Strip Chamber (CSC) is installed outside the magnet. The CSC is used for improvement of particles momentum identification. The structure of the GEM detectors and the CSC prototype and the results of study of their characteristics are presented. The GEM detectors and CSC are integrated into the BM@N experimental setup and data acquisition system. The results of first tests of the GEM tracking system and CSC in last runs are shortly reviewed.
\end{abstract}

\section{Introduction}

Collisions of Nuclotron heavy ion beams with fixed targets provide a unique opportunity to study strange mesons and multi-strange hyperons close to the kinematic threshold. One of the main goals of the experiment is to measure yields of light hyper-nuclei, which are expected to be produced in coalescence of $\Lambda$-hyperons with nucleons.

\section{BM@N experimental setup}

A scheme of the BM@N experimental setup is shown in Fig. 1. The experiment combines high-precision track measurements with the time-of-flight information for particle identification and calorimetry for the analysis of the collisions centrality. The magnetic field of the analyzing magnet can be varied up to $1 \mathrm{~T}$ to get the optimal detector acceptance and momentum resolution for different reactions and beam energies. The charged particle momentum and multiplicity are measured with the set of Gas Electron Multipliers (GEM) located inside the analyzing magnet and by Drift chambers (DCH) situated outside the magnetic field. Double-sided silicon strip-detectors (SiMS) are installed between the GEM tracker and the target to improve the track and vertex reconstruction in heavy-ion collisions. A Cathode Strip Chamber (CSC) is installed outside the magnet between GEM detectors and ToF400.

\footnotetext{
*e-mail: Elena.Kulish@cern.ch
} 


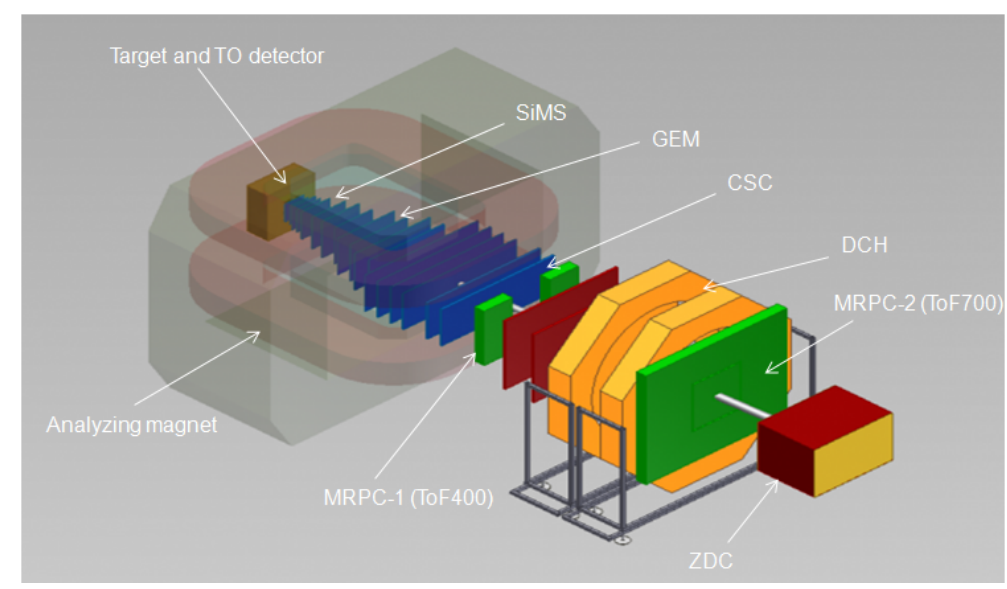

Figure 1. Schematic view of the BM@N experimental setup: SiMS - Silicon Micro-strip Sensors, GEM - Gas Electron Multipliers, CSC - Cathode Strip Chamber (CSC), mRPC - Multi-gap Resistive Plate Chambers, DCH - Drift Chambers, ZDC - Zero Degree Calorimeter

\section{GEM tracking system}

The tracking system of the BM@N experiment will provide precise momentum measurements of the cascade decays products of multi-strange hyperons and hyper-nuclei produced in central $\mathrm{Au}-\mathrm{Au}$ collisions. All physics measurements will be performed in conditions of high beam intensities in collisions with large multiplicity of charged particles. This requires the use of detectors with the capacity to resolve multi tracks produced at a very high rate.

The basic requirements for the tracking system are:

- capability of stable operation in conditions of high loadings up to $10^{5} \mathrm{~Hz} / \mathrm{cm}^{2}$;

- spatial resolution better than $300 \mu \mathrm{m}$ in a magnetic field of $0.8 \mathrm{~T}$;

- geometrical efficiency better than $95 \%$;

- maximum possible geometrical acceptance within the BM@N experiment dimensions;

- capability to function in a $0.8 \mathrm{~T}$ magnetic field.

For this reason, two coordinate GEM detectors were chosen for the central part of the tracking system. At present, the GEM tracking system of the BM@N experiment consists of twelve detectors: five GEM detectors of a size of $66 \times 41 \mathrm{~cm}^{2}$ (small GEM detectors) and seven GEM detectors of a size of $163 \times 45 \mathrm{~cm}^{2}$ (big GEM detectors). These detectors were installed into the BM@N setup for technical runs with the deuteron, carbon, argon and krypton beams in 2016-2018. The GEM detector technology and construction are described in detail in [1].

\section{Cathode strip chamber}

The Cathode Strip Chamber (CSC) is intended to precise parameters of tracks obtained in GEM detectors inside the analyzing magnet. Beside improvement of particles momentum identification, a refined track in CSC is used to find the corresponding hit in the time-of-flight system (ToF400). 


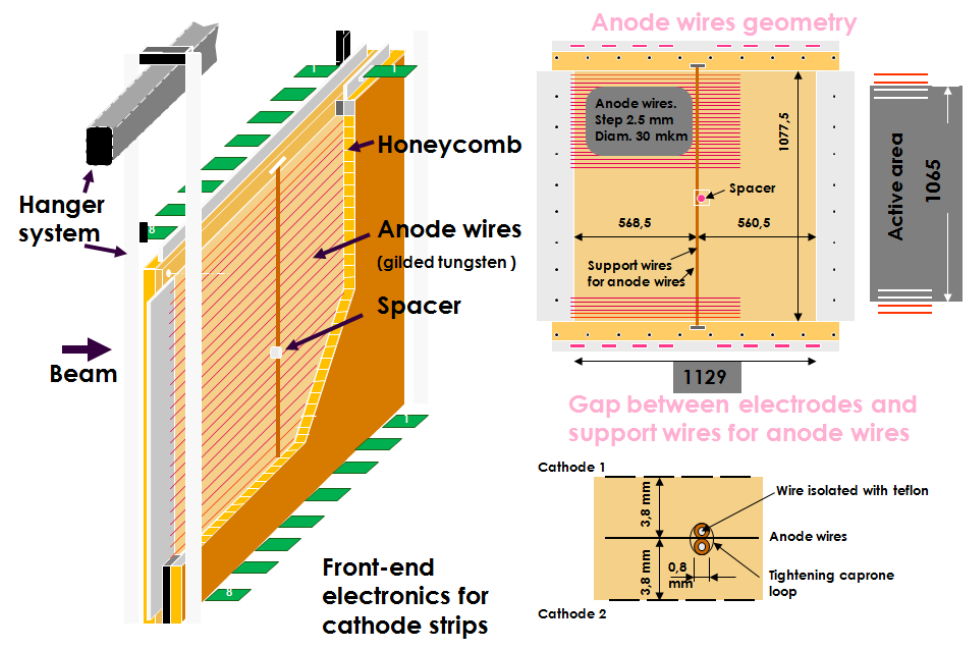

Figure 2. Schematic view of Cathode Strip Chamber (CSC)

A schematic view of the CSC is shown in Fig. 2. The cathode strip chamber consists of the anode plane and two cathode planes. The anode plane is a set of gilded tungsten wires with a diameter of $30 \mu \mathrm{m}$ and a step of $2.5 \mathrm{~mm}$. The gap between the anode plane and each cathode plane is $3.8 \mathrm{~mm}$. There is a spacer between two cathodes to prevent deformation of the chamber due to the gas filling. To fix the anode wire raw two support wires are used. The size of the active area of the chamber is $1129 \times 1065 \mathrm{~mm}^{2}$.

Both cathodes are made of printed circuit boards. A two-coordinate readout of the signal is performed on two cathode boards with sets of parallel metal strips. The inclination angles of cathode strips to the vertical axis are 0 (X coordinate) and 15 (Y coordinate) degrees. The widths of the $\mathrm{X}$ and $\mathrm{Y}$ strips are the same and equal to $2.5 \mathrm{~mm}$. The PCB are glued to support the honeycomb. The number of vertical and inclined strips is 1808 and 1928, respectively.

The cathode strip chamber is developed and assembled in JINR.

\section{FEE and DAQ}

Both the GEM and the CSC front-end electronics is based on the charge sensitive preamplifier chip VA162 and modified chip VA163 (IDEAS) [2]. The chip has 32 channels. Each channel contains a charge sensitive preamplifier, a shaper with $2 \mu$ s peaking time for VA162 and 500 ns for VA163 and a sample-hold circuit (see Tab. 1). An analog multiplexer with 32 inputs allows one to perform a serial readout channel by channel.

The chips are joined in groups of 4 in one front-end board. The multiplexed data from each board are transmitted through $13 \mathrm{~m}$ of a twisted pair flat cable to a 12-bit analog-todigital converter (ADC) readout by the BM@N data acquisition system [3].

\section{Beam results}

To separate positively and negatively charged particles and to measure their momentum the central tracking system is located inside the analyzing magnet. Since the magnetic field is perpendicular to the electric field direction in the GEM, the electron avalanche in GEM 
Table 1. VA162 and VA163 characteristics

\begin{tabular}{lll}
\hline & VA162 & VA163 \\
\hline Number of channels & 32 & 32 \\
Input charge & $-1.5 \mathrm{pC} \div+1.5 \mathrm{fC}$ & $-750 \mathrm{fC} \div+750 \mathrm{fC}$ \\
Shaping time & $2 \div 2.5 \mu \mathrm{s}$ & $500 \mathrm{~ns}$ \\
Noise & $2000 \mathrm{e}$ ENC at $50 \mathrm{pF}$ load & $1797 \mathrm{e}$ ENC at $120 \mathrm{pf}$ load \\
Linearity positive charge & $1 \%$ & $0.5 \%$ \\
Linearity negative charge & $3 \%$ & $1.4 \%$ \\
Gain & $0.5 \mu \mathrm{A} / \mathrm{fC}$ & $0.88 \mu \mathrm{A} / \mathrm{fC}$ \\
Total power max. & $66 \mathrm{~mW}$ & $77 \mathrm{~mW}$ \\
\hline
\end{tabular}

gas gaps drifts at some angle (the Lorentz angle) with respect to the normal to the readout board. The electron drift results in a displacement of the collected charge called a Lorentz shift. The shift value depends on the field strength and gas properties. In order to eliminate a systematic shift of the reconstructed tracks in the magnetic field, the GEM detectors are rotated so that neighboring planes have opposite directions of the electric field. To ensure effective operation, high spatial resolution and to reduce both the Lorentz shift value and the probability of discharge the GEM tracking system performance was tested for different gas mixtures and electric field values in gas gaps. First beam tests of GEM chambers were performed in 2016 with the deuteron beam at the Nuclotron accelerator. The GEM chambers were filled with an $\operatorname{Ar}(70) / \mathrm{CO}_{2}(30)$ gas mixture. To measure the spatial accuracy of the GEM detectors the distributions of hit deviations from the reconstructed tracks (the hit residuals) were calculated. The obtained standard deviation of the Gaussian fit is $\sim 670 \mu \mathrm{m}$ (see Fig. 3, left).

At the Ar and $\mathrm{Kr}$ beam tests in 2018 the value of the electric field in drift gaps of the GEM chambers was increased to $1.5 \mathrm{kV} / \mathrm{cm}$, the gas mixture was changed to the $\operatorname{Ar}(80) / \mathrm{C}_{4} \mathrm{H}_{10}(20)$. That caused the decrease of the Lorentz shift value of the electrons avalanche. The hit residuals standard deviation of the Gaussian fit is $\sim 220 \mu \mathrm{m}$ (see Fig. 3, right).
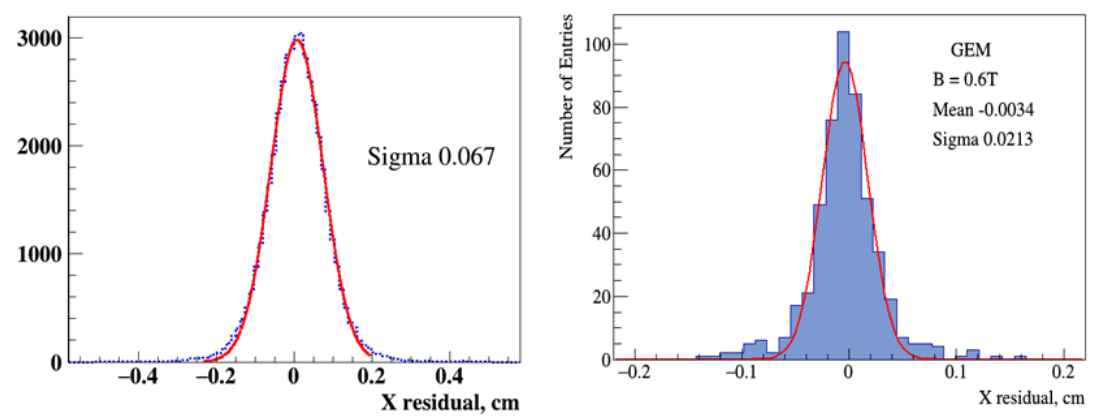

Figure 3. GEM hit residuals for a magnetic field of $0.6 \mathrm{~T}$ for different gas mixtures

\section{Beam tests CSC}

The first tests of the CSC prototype were performed in a technical run in the BM@N in February-March 2018 at the Ar and Kr Nuclotron beams. The chamber was installed in front 
of ToF400 to check its performance as an outer tracker for heavy ions. The CSC chamber was filled with a flowed $\operatorname{Ar}(75) / \mathrm{C}_{4} \mathrm{H}_{10}(25) / \mathrm{C}_{3} \mathrm{H}_{8} \mathrm{O}$ gas mixture and equipped with the VA162 (in Ar beam run) and VA163 (in Kr beam run) based electronics. The recorded ADC data undergo the pedestal subtraction procedure. The clusters are determined as groups of adjacent strips with amplitudes of signals above the threshold. For reconstructed clusters, the center of gravity, the width and the total charge are calculated. The average cluster width is shown in Fig. 4, left. After applying the alignment procedure for the CSC position, the cluster data were converted into the spatial coordinates of hits. The tracks of charged particles were reconstructed in the GEM central tracking system and extrapolated into the CSC. The track detection efficiency of the CSC chamber was calculated. The efficiency of the chamber was determined from the presence of a cluster with a center of gravity within $\pm 1 \mathrm{~cm}$ of a predicted position. The efficiency distribution over the chamber surface is presented in Fig. 4, right. The distributions of CSC hit deviations from the reconstructed tracks were calculated within a magnetic field switched off and a magnetic field of $0.6 \mathrm{~T}$ (see Figs. 5 and 6).
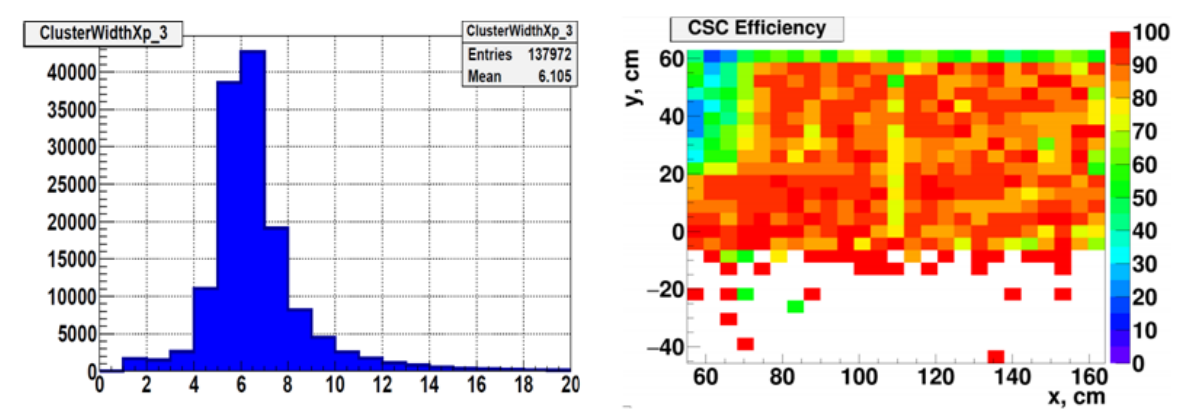

Figure 4. Cluster width for the CSC (left). Efficiency distribution (right)
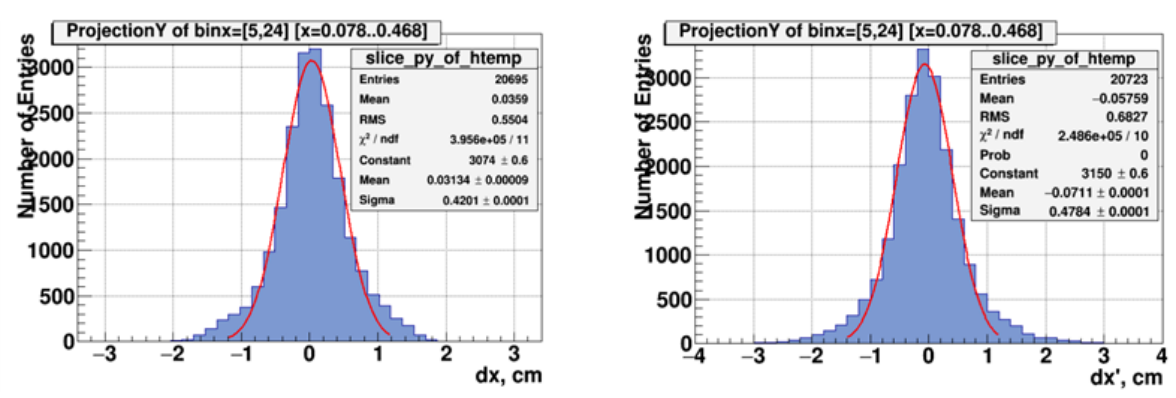

Figure 5. CSC X and Y hit residuals: magnetic field switched off

\section{Conclusion}

Triple GEM detectors of the BM@N tracking system have been assembled and studied in the d, C, Ar, Kr beams of the Nuclotron accelerator. The measured parameters of the GEM detectors are consistent with the design specifications. Seven GEM chambers with a size of $1632 \mathrm{~mm} \times 450 \mathrm{~mm}$ are the biggest GEM detectors presently produced in the world. 

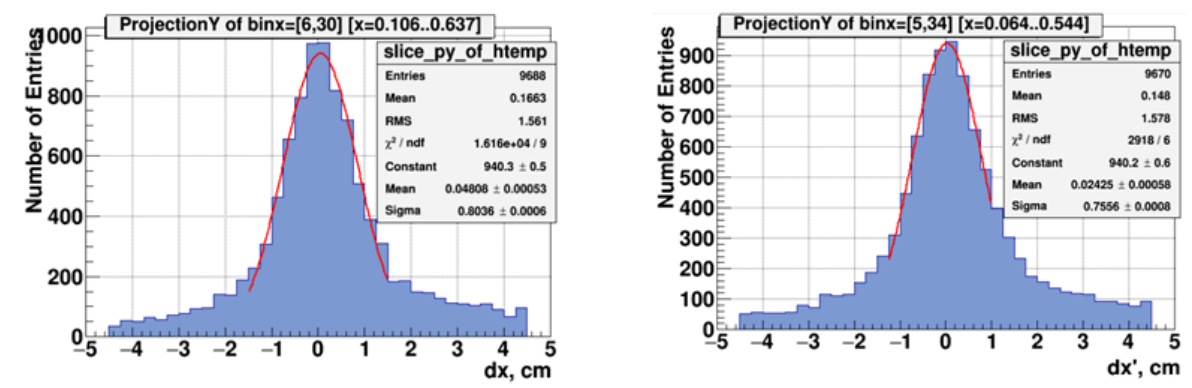

Figure 6. CSC X and Y hit residuals: magnetic field of $0.6 \mathrm{~T}$

For today the GEM tracking system is:

- 12 chambers $660 \times 412 \mathrm{~mm}^{2}$ (5) and $1632 \times 450 \mathrm{~mm}^{2}(7)$,

- $\sim 6.5 \mathrm{~m}^{2}$ active area,

- $\sim 1$ billion of independent amplification channels,

- $\sim 45000$ strips/electronics channels,

- $>3 \mathrm{~km}$ of control and readout cables.

The first prototype of the CSC was tested in a technical run of the BM@N in February-March 2018. Characteristics are still under investigation. However, the first results showed that the chamber functions properly.

\section{References}

[1] D. Baranov et al., Journal of Instrumentation 12, C06041 (2017)

[2] http://ideas.no/products/ide1162/

[3] https://afi.jinr.ru/ 